\title{
Interdomain Dynamics Explored by Paramagnetic NMR
}

\author{
Luigi Russo, $^{\dagger}$ Mitcheell Maestre-Martinez, ${ }^{\dagger}$ Sebastian Wolff, Stefan Becker, and Christian Griesinger*
}

NMR Based Structural Biology, Max Planck Institute for Biophysical Chemistry, Am Fassberg 1137077 Göttingen, Germany

Supporting Information

ABSTRACT: An ensemble-based approach is presented to explore the conformational space sampled by a multidomain protein showing moderate interdomain dynamics in terms of translational and rotational motions. The strategy was applied on a complex of calmodulin ( $\mathrm{CaM})$ with the IQ-recognition motif from the voltage-gated calcium channel $\mathrm{Ca}_{\mathrm{v}} 1.2$ (IQ), which adopts three different interdomain orientations in the crystal. The N60D mutant of calmodulin was used to collect pseudocontact shifts and paramagnetically induced residual dipolar couplings for six different lanthanide ions. Then, starting

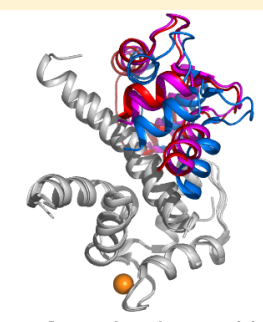

Conformational ensemble in the crystal

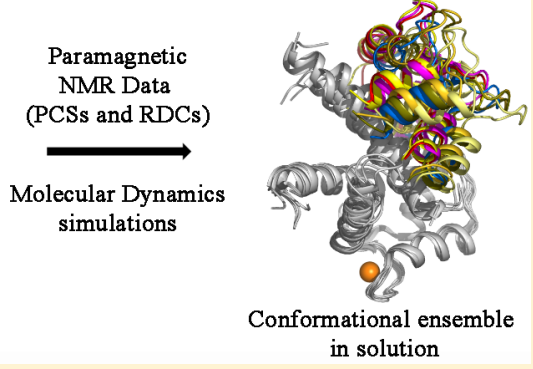
from the crystal structure, pools of conformations were generated by free MD. We found the three crystal conformations in solution, but four additional MD-derived conformations had to be included into the ensemble to fulfill all the paramagnetic data and cross-validate optimally against unused paramagnetic data. Alternative approaches led to similar ensembles. Our "ensemble" approach is a simple and efficient tool to probe and describe the interdomain dynamics and represents a general method that can be used to provide a proper ensemble description of multidomain proteins.

\section{INTRODUCTION}

Interdomain motions within multidomain proteins play a crucial role in many biological processes such as catalysis, channel activation, and molecular recognition. ${ }^{1-5}$ This motion affects both the orientation of the domains with respect to each other as well as the conformation of each of the domains and can be described by ensembles. Population shifts of the ensemble members are observed when the proteins interact with their targets. The functional importance of interdomain dynamics has led to a growing interest in the development of methods to characterize this type of motion.

The complete description of protein dynamics requires the full characterization of the ensemble of conformations adopted by the protein, with all the rates of interconversion between them determined. Several experimental methods are currently being employed to derive ensembles of multidomain proteins. X-ray crystallography reports conformational plasticity of multidomain proteins if the protein adopts different structures in the unit cell or in different crystal forms. ${ }^{6}$ Nevertheless, crystal packing may distort the conformational ensemble from the ensemble observed in solution. ${ }^{7-9}$ Nuclear magnetic resonance (NMR) spectroscopy is a powerful tool for studying molecular motions ${ }^{10-19}$ of proteins in solution. Domain dynamics has an effect on the diffusion tensor of the protein which can be used to characterize the domain motion. ${ }^{13,14,20-24}$ However, such approaches suffer from the necessity to model the effect of the domain motion on the diffusion tensor, which is model-dependent. Residual dipolar couplings (RDCs) have been successfully used for the description of dynamics within protein domains. Nevertheless, domain dynamics alters the alignment tensors, and it is complicated to disentangle the domain motion from the alignment tensor modulation. This requires accurate modeling as has been recently pointed out again. ${ }^{25}$ Using residual dipolar couplings from alignment media has recently been shown to deliver biased results on the example of the small biomolecules lactose and chitobiose. In these examples, RDCs derived from measurements in alignment media ${ }^{26}$ did not reproduce the ensembles derived from paramagnetic alignment ${ }^{27,28}$ which most reliably detects domain motion as described below. On the other hand, if the alignment tensor generated by alignment media can be calculated, conformational ensembles can be determined by so-called multiple alignment tensor fits as has been demonstrated recently on the example of a flexible small molecule. $^{29}$

Qualitatively completely different information without these shortcomings is provided by anisotropic NMR parameters that are induced by self-alignment of paramagnetic or at least magnetically anisotropic biomolecules. Such self-alignment can be achieved by binding of paramagnetic metal ions, mostly lanthanides, to natural binding sites of the biomolecule ${ }^{30}$ or to small molecule chemical metal chelating tags attached to the biomolecule. ${ }^{31-51}$ The paramagnetically induced alignment of the domain with the lanthanide (tagged) is then different from the alignment induced in the domains (nontagged) of the protein unless the tagged and nontagged domains do not move with respect to each other. ${ }^{30}$ The alignment tensors of the tagged and nontagged domains can be determined from the measurement of the RDCs in the respective domains. In

Received: August 6, 2013

Published: October 11, 2013 
addition to RDCs, the pseudocontact shifts $(\mathrm{PCSs})^{30}$ are modulated by the domain reorientation (also measured by the RDCs) and also by the domain translation. The combined use of RDCs and PCSs obtained with paramagnetic ions of different alignment strengths provides a unique way to describe complex mixtures of translational and rotational interdomain motions. ${ }^{52-55}$ So far, it has been difficult to extract concrete ensembles of multidomain proteins from paramagnetic data, partially because the systems chosen were very dynamic, such as calmodulin $(\mathrm{CaM})$. In these cases, concepts of maximum occurrence were developed. ${ }^{52,53,56-58}$ For conformationally more restricted cases such as the TAR-RNA ensemble, approaches have been employed that produced ensembles which closely matched a set of crystal structures of the same RNA with target molecules. ${ }^{55}$ Here, we investigate the calmodulin complex with the IQ-recognition motif from the voltage-gated calcium channel $\mathrm{Ca}_{\mathrm{v}} 1.2$ (IQ), which adopts three conformations in the crystal. ${ }^{59}$ Using paramagnetic data from six different lanthanides generated with the previously developed CaMN60D mutant, ${ }^{60}$ we studied the conformational space of this complex in solution using an ensemble-based approach. Our results confirm the existence of the three crystal conformations in solution and also show that four additional conformations have to be included into the ensemble to fulfill all the paramagnetic data and cross-validate optimally against unused paramagnetic data. This is in contrast with a previous publication on similar $\mathrm{CaM} /$ target complexes, in which crystal structures were refined using paramagnetic data, using single models to describe all the data. ${ }^{61}$ The protocol developed here for the description of interdomain dynamics is of general nature for ensemble description of multidomain proteins.

\section{MATERIALS AND METHODS}

Protein Expression and Purification. ${ }^{15} \mathrm{~N},{ }^{13} \mathrm{C}$-labeled CaMN60D was expressed and purified following a published protocol. ${ }^{62}$ The coding sequence for the IQ peptide was cloned into a modified pET28a vector (Novagen) coding for an $\mathrm{N}$-terminal $\mathrm{Z}$ tag fusion protein. ${ }^{63}$ The fusion protein was expressed in rich medium at $22{ }^{\circ} \mathrm{C}$. After cell lysis, the protein was bound to Ni-NTA resin (Qiagen). Subsequently, the resin-bound fusion protein was incubated with excess ${ }^{15} \mathrm{~N},{ }^{13} \mathrm{C}$-labeled CaMN60D. The complex of the IQ peptide with ${ }^{15} \mathrm{~N},{ }^{13} \mathrm{C}$-labeled CaMN60D was released from the resin by cleavage with tobacco etch virus (TEV) protease. The released complex was further purified by anion exchange chromatography on a Sepharose Q HP column (GE Healthcare) in the presence of $1 \mathrm{mM}$ $\mathrm{CaCl}_{2}$ to obtain highly purified ${ }^{15} \mathrm{~N}^{13} \mathrm{C} \mathrm{Ca}_{4} \mathrm{CaMN60D/IQ}$ complex.

Sample Preparation. The ${ }^{15} \mathrm{~N},{ }^{13} \mathrm{C} \mathrm{Ca}_{4} \mathrm{CaMN60D} / \mathrm{IQ}$ complex was dialyzed against $20 \mathrm{mM}$ Bis-Tris (pH 6.8), $150 \mathrm{mM} \mathrm{KCl}$, and 125 $\mu \mathrm{M} \mathrm{CaCl}$. NMR samples of the complex were prepared at a final protein concentration of $0.5 \mathrm{mM}$ in the same buffer supplemented with $10 \%{ }^{2} \mathrm{H}_{2} \mathrm{O}$. In order to substitute the calcium ion in the second metal binding loop of the $\mathrm{N}$-terminal domain by paramagnetic lanthanides, the ${ }^{15} \mathrm{~N},{ }^{13} \mathrm{C} \mathrm{Ca}_{4} \mathrm{CaMN} 60 \mathrm{D} / \mathrm{IQ}$ samples were titrated with $10 \mathrm{mM}$ solutions of analytical grade $\mathrm{LnCl}_{3}(\mathrm{Ln}=\mathrm{Tb}, \mathrm{Dy}, \mathrm{Ho}, \mathrm{Er}, \mathrm{Tm}$, $\mathrm{Yb}$ ) (Sigma Aldrich). The titrations were performed by following the ${ }^{1} \mathrm{H},{ }^{15} \mathrm{~N}$ HSQC spectra.

NMR Spectroscopy. The NMR experiments were carried out on a $700 \mathrm{MHz}$ AVANCE-III Bruker spectrometer equipped with a triple resonance probe and on a $900 \mathrm{MHz}$ spectrometer with a triple resonance $5 \mathrm{~mm}$ cryoprobe with a $z$-axis pulse field gradient. All spectra were acquired at $303 \mathrm{~K}$. To obtain sequence-specific backbone resonance assignments, $\mathrm{HNCA}^{64-66}$ and $\mathrm{CBCA}(\mathrm{CO}) \mathrm{HN}^{67}$ spectra were recorded at $700 \mathrm{MHz}$. The assignment of the lanthanide-loaded samples was confirmed by inspection of the ${ }^{1} \mathrm{H},{ }^{15} \mathrm{~N}$ HSQC and HNCA spectra of paramagnetic and diamagnetic samples. The recycle delays were in the range of $0.7 \mathrm{~s}$ (paramagnetic) and $1.5 \mathrm{~s}$ (diamagnetic).
Lanthanum was used as diamagnetic reference as it has an ionic radius similar to those of the paramagnetic lanthanides. PCSs were measured as the difference between the chemical shift of corresponding nuclei in the paramagnetic and diamagnetic samples. Residual dipolar couplings ${ }^{1} D_{\mathrm{HN}}$ were measured at $900 \mathrm{MHz}$ as the ${ }^{15} \mathrm{~N}$-doublet splitting of the paramagnetic sample minus that of the diamagnetic sample using the IPAP pulse sequence. ${ }^{68}$ In all experiments, the concentration of ${ }^{15} \mathrm{~N},{ }^{13} \mathrm{C}$-labeled CaMN60D was about $0.5 \mathrm{mM}$ with a slight excess of unlabeled IQ peptide.

All NMR spectra were processed with the software NMRpipe. ${ }^{69}$ The programs SPARKY ${ }^{70}$ and CARA ${ }^{71}$ were used for analysis of the 2D and $3 \mathrm{D}$ spectra, respectively.

Protein Coordinates and Reference System. The current study was performed using the coordinates from the three X-ray structures of the $\mathrm{CaM} / \mathrm{IQ}$ complex referred to as models $\mathrm{A}, \mathrm{B}$, and C (PDB code 2BE6) and models generated by molecular dynamics (MD) simulations at $300 \mathrm{~K}$ applying the amber99sb force field. ${ }^{72}$ The simulations were carried out in GROMACS, ${ }^{73}$ using the standard parameters delivered by the authors of the software. In total, two MD simulations of $120 \mathrm{~ns}$ were performed, starting from either model A or $\mathrm{C}$, and the coordinates were recorded every 5 ps for a total of 48000 MD models (24 000 from each simulation). All structural models were aligned on the model $\mathrm{A}$ from the $\mathrm{X}$-ray structure. In evaluations regarding the $\mathrm{N}$-terminal domain and the interdomain dynamics, the models were aligned on the backbone of the $\mathrm{N}$-terminal domain (i.e., residues 5-74). For evaluations of the C-terminal domain data, the structures were aligned with respect to the backbone of residues $82-$ 146. The CaM backbone coordinates of the best set of MD models are provided in the Supporting Information. The structures were visualized and evaluated by using the programs PyMOL, ${ }^{74}$ PROCHECK-NMR, ${ }^{75}$ and MolProbity. ${ }^{76}$

Evaluation of Paramagnetic Data. PCS of ${ }^{\mathrm{N}} \mathrm{H}, \mathrm{N}$ nuclei and $\mathrm{RDC}$ of the ${ }^{\mathrm{N}} \mathrm{H}-\mathrm{N}$ pairs were evaluated with a series of in-house written Python or Matlab scripts. PCS and RDC tensors were calculated by least-squares fits of the experimental values to the protein coordinates, using the equations ${ }^{30}$

$$
\begin{aligned}
\delta_{i}^{\mathrm{PCS}}, \Delta v_{i}^{\mathrm{RDC}} & =k \cdot \operatorname{trace} \times\left[\begin{array}{ccc}
\left(3 x_{i}^{2}-r_{i}^{2}\right) & 3 x_{i} y_{i} & 3 x_{i} z_{i} \\
3 x_{i} y_{i} & \left(3 y_{i}^{2}-r_{i}^{2}\right) & 3 y_{i} z_{i} \\
3 x_{i} z_{i} & 3 z_{i} z_{i} & \left(3 z_{i}^{2}-r_{i}^{2}\right)
\end{array}\right) \\
& \left.\left(\begin{array}{ccc}
\chi_{x x} & \chi_{x y} & \chi_{x z} \\
\chi_{x y} & \chi_{y y} & \chi_{y z} \\
\chi_{x z} & \chi_{y z} & \chi_{z z}
\end{array}\right)\right]
\end{aligned}
$$

where

$$
k_{\mathrm{PCS}}=\frac{1}{12 \pi r_{i}^{5}}, \quad k_{\mathrm{RDC}}=\frac{B_{0}^{2} \gamma_{\mathrm{H}} \gamma_{N} \hbar}{120 \pi^{2} k_{B} T r_{i}^{5}}
$$

$r_{i}$ is either the distance to the paramagnetic center (for PCSs) or the length of the $\mathrm{N}-{ }^{\mathrm{N}} \mathrm{H}$ bond (for RDCs), and the $(x, y, z)$ coordinates also refer to these vectors. For all evaluations with structural ensembles, we first evaluated the nonlinear coordinate-dependent terms (i.e., the terms describing the vector orientation and the $r^{3}$ terms in the case of PCS) for all conformers, and then we averaged them and performed the least-squares fits of the tensors. In the PCSs' evaluation, the metal position was taken from the PDB coordinates of the X-ray structure in the case of the $\mathrm{N}$-terminal domain, that is, the position of the second calcium ion. A grid search around this metal position was also conducted to investigate whether this position was optimal. For the C-terminal domain, the metal position was determined from PCS values using a grid search algorithm until the metal coordinates that provided the smallest $Q$ factor were found. The simultaneous evaluation of PCS and RDC was performed similar to the PCS evaluation, with the additional incorporation of the RDCs in the fit. In 
this case, in order to avoid overestimation of any of the two parameters, the values of both data sets were scaled with the largest PCS and RDC values, respectively. Additionally, we minimized a composite $Q$ factor that is a combination of the PCS and RDC $Q$ factors, using the following equation:

$$
Q_{\mathrm{PCS}+\mathrm{RDC}}=\sqrt{\frac{\left(N_{\mathrm{RDC}} Q_{\mathrm{RDC}}^{2}+N_{\mathrm{PCS}} Q_{\mathrm{PCS}}^{2}\right)}{\left(N_{\mathrm{RDC}}+N_{\mathrm{PCS}}\right)}}
$$

where $N$ is the number of respective parameters used to determine the $Q$ factors. $Q$ factors were calculated as proposed by Cornilescu et al. ${ }^{77}$ For the estimation of errors in $Q$ factors, tensors, and metal positions, we used a boot-strapping method, where 100 data sets composed of $80 \%$ of the experimental data points were randomly generated and then subsequently evaluated.

Evaluation of Interdomain Dynamics. In order to assess the interdomain dynamics, we used a model-based approach, which relies on the crystal structure and the addition of conformations selected from free molecular dynamics runs. The optimal ensemble of conformations was found in the following way: (a) evaluation of the tensor from the $\mathrm{N}$-terminal domain ensemble using RDCs and PCSs; (b) prediction of RDCs and PCSs for the different models and subsequent averaging of these parameters for the ensemble at equal populations; (c) selection of the ensemble providing the lowest Cterminal $Q$ factor (eq 2); (d) optimization of the populations that minimize the $Q$ factor (optional). Details of the protocol for the incorporation of MD models in the evaluation are provided in the Supporting Information.

Validation of CaM/DRP-1 and CaM/DAPk Complexes. For the tensor evaluation performed on these systems, we used the protein coordinates from the PDB files 2K0J (CaM/DRP-1) and 2K61 (CaM/ $\mathrm{DAPk})$, and the corresponding published PCS and RDC data. ${ }^{61}$ In the same manner as we did with the CaM/IQ complex, we evaluated the $\mathrm{N}$-terminal domain using the residues $5-74$ and the C-terminal domain using the residues $82-146$. The RDCs from mobile residues, listed in the Supporting Information of the original publication, ${ }^{61}$ were also discarded in our evaluation.

\section{RESULTS AND DISCUSSION}

Collection of the Paramagnetic-Based Restraints. Paramagnetic data were obtained using the CaMN60D mutant, in which the second calcium binding site of the $\mathrm{N}$-terminal domain binds selectively a lanthanide ion. ${ }^{60}$ The N60D mutation and the lanthanide substitution do not lead to any significant structural changes in the region flanking the metal coordination site. ${ }^{52,60,78}$ PCSs of $\mathrm{H}^{\mathrm{N}}$, N nuclei, and RDCs of the $\mathrm{H}^{\mathrm{N}}-\mathrm{N}$ pairs for six paramagnetic lanthanide $(\mathrm{CaLn})_{\mathrm{N}}\left(\mathrm{Ca}_{2}\right)_{\mathrm{C}} \mathrm{CaMN60D}$ forms in complex with IQ peptide $(\mathrm{Ln}=\mathrm{Tb}, \mathrm{Dy}, \mathrm{Ho}, \mathrm{Er}, \mathrm{Tm}, \mathrm{Yb})$ were measured using ${ }^{1} \mathrm{H},{ }^{15} \mathrm{~N}$ HSQC and IPAP spectra, respectively (Figure 1).

Additionally, the diamagnetic reference spectra were measured using a lanthanum-loaded $(\mathrm{CaLa})_{\mathrm{N}}$ $\left(\mathrm{Ca}_{2}\right)_{\mathrm{C}} \mathrm{CaMN60D/IQ}$ complex. A complete assignment of the observable $\mathrm{NH}$ backbone amide resonances has been obtained using standard triple resonance experiments (see the Materials and Methods section) for all the lanthanide derivate samples. The resonances of the residues within the shell around the paramagnetic center, whose radius depends on the nuclear relaxing capability of the metal ion, on the electron relaxation time, and on the rotational correlation time of the molecule, were broadened beyond detection due to paramagnetic relaxation enhancement (PRE). So far, for terbium (the lanthanide with the largest Curie spin), a total of 59 measurable RDCs (102 PCSs) were collected, while for ytterbium (the paramagnetic lanthanide used with the smallest Curie spin), 74 RDCs (119 PCSs) were measured. Moreover, the RDCs of the
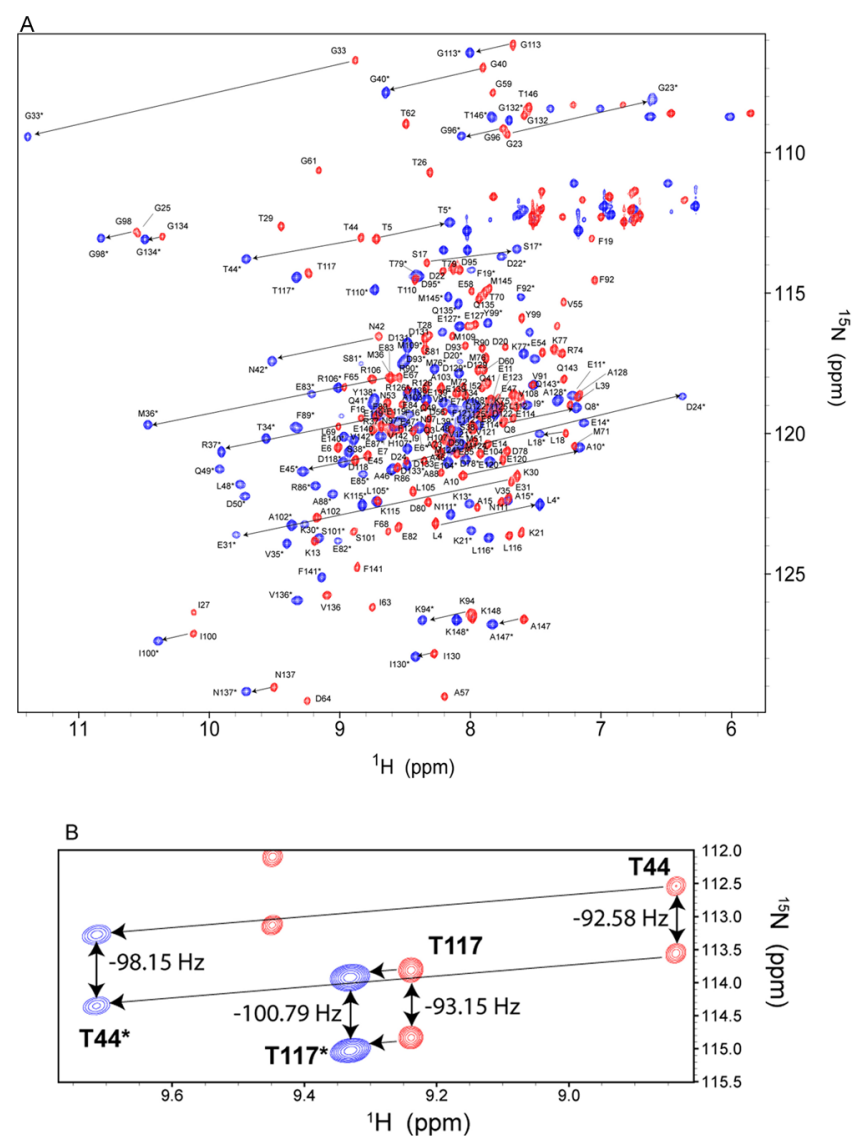

Figure 1. NMR experiments. (A) ${ }^{1} \mathrm{H},{ }^{15} \mathrm{~N}$ HSQC spectrum recorded at $303 \mathrm{~K}$ at pH 6.8 using a $700 \mathrm{MHz}$ NMR spectrometer for CaMN60D/ IQ complex loaded with diamagnetic lanthanum (CaMN60D$(\mathrm{CaLa})_{\mathrm{N}}\left(\mathrm{Ca}_{2}\right)_{\mathrm{C}} / \mathrm{IQ}$ red) and paramagnetic thulium (CaMN60D$(\mathrm{CaTm})_{\mathrm{N}}\left(\mathrm{Ca}_{2}\right)_{\mathrm{C}} / \mathrm{IQ}$ blue). (B) Selected spectral region of ${ }^{1} \mathrm{H},{ }^{15} \mathrm{~N}$ HSQC IPAP.

residues that showed fast motions in the picosecondnanosecond time scale with order parameters below 0.8 , as reported for other $\mathrm{CaM} /$ peptide complexes (CaM-KKalpha (BMRB 15183), CaM/K1p (BMRB 15184), CaM/eNOSp (BMRB 15185), CaM/smMLCKp (BMRB 15186), CaM/ PDEs (BMRB 15187), and CaM/nNOSp (BMRB 15191)), ${ }^{79}$ were not used as constraints in the evaluation. The following analysis has been performed using a substantial number of PCSs and RDCs for all lanthanide data sets (Tables SI1, SI2, and SI3).

Individual Fitting of the Data to the N-Terminal and C-Terminal Domains Indicates Motions within Each of the Domains. In order to check the quality of the experimental data, we fitted the RDCs and PCSs to each of the three X-ray models A, B, and C. To remove any influence from the interdomain arrangement in the analysis, the fits were performed using each CaM domain separately for each of the metals. The PCSs provided excellent fits for both CaM domains (Figure SI1) as reflected by $Q$ factor values in the range of $0.03-0.11$ for the N-terminal domain and $0.09-0.17$ for the Cterminal domain. Also, the RDCs showed good quality fits, with $Q$ factors ranging from 0.13 to 0.31 and 0.18 to 0.32 for the $\mathrm{N}$ and C-terminal domains, respectively. In the case of the $\mathrm{N}$ terminal domain, the one that carries the lanthanides, the magnetic susceptibility tensors calculated for either PCS or $\mathrm{RDC}$ data sets are very similar (Table SI4), indicating that the 

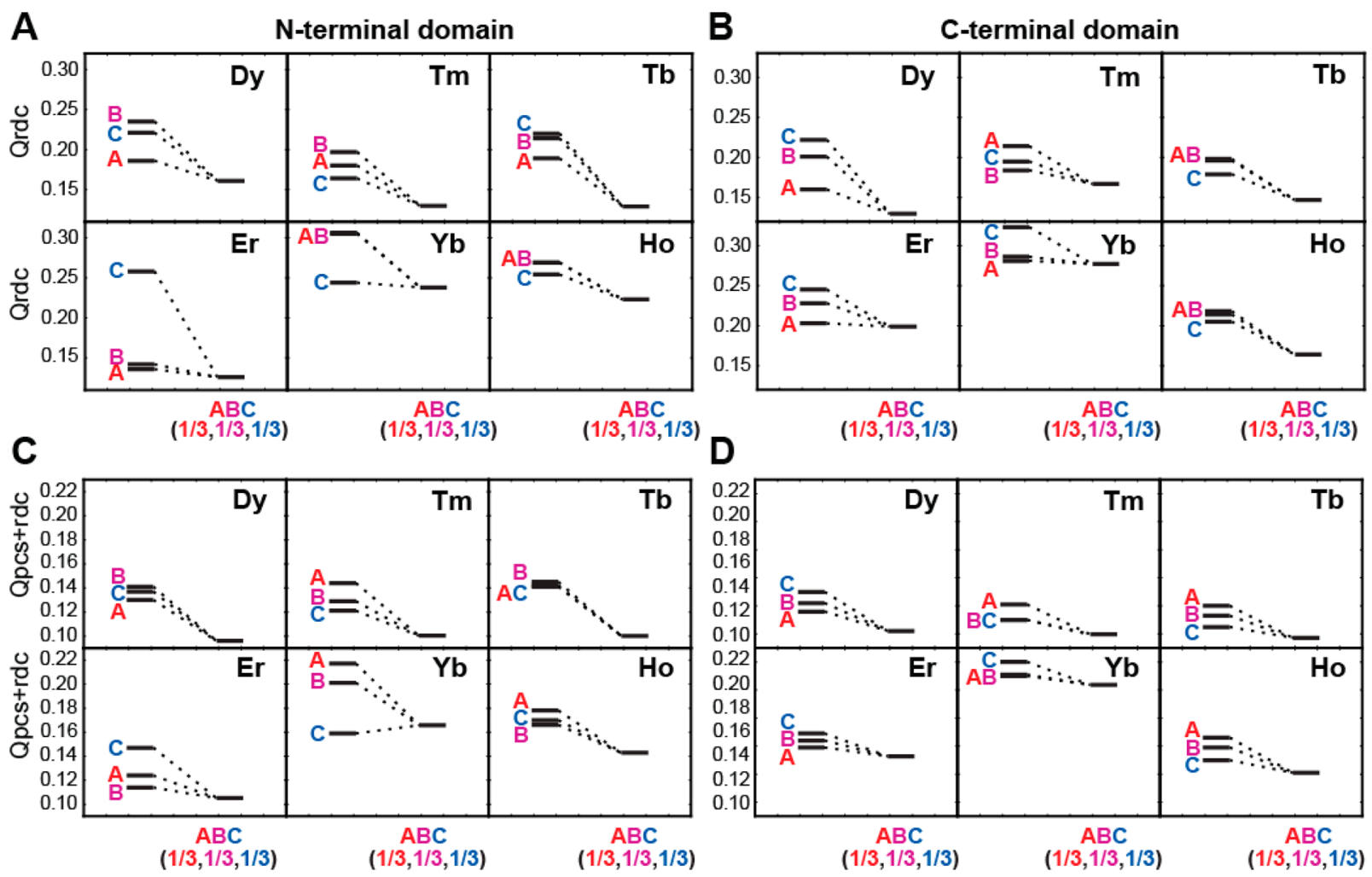

Figure 2. Comparison of the $Q$ factors for the fit of the data to the individual domains. Fitting of RDC or PCS+RDC data individually to the structure of either $\mathrm{N}-(\mathrm{A}, \mathrm{C})$ or $\mathrm{C}$-terminal $(\mathrm{B}, \mathrm{D}) \mathrm{CaM}$ domains assuming single structures or an ensemble of equally populated conformations (models A-B-C).

metal is rigidly bound to the protein and the tensor does not wobble. If the tensor wobbled, the RDC-derived tensor would be smaller than the PCS-derived tensor due to the higher sensitivity of RDCs to local motions ${ }^{80}$ compared to PCSs. Consequently, we could fit the PCS and RDC data together using a single tensor (i.e., PCS+RDC fit), which does not increase the $Q$ factors for either data set (Tables SI4 and SI5) considerably. Additionally, we also performed this evaluation optimizing the lanthanide position, in order to take into account any possible difference in the geometry of the coordination sphere between $\left(\mathrm{Ca}_{2}\right)_{\mathrm{N}}\left(\mathrm{Ca}_{2}\right)_{\mathrm{C}} \mathrm{CaM}$ and $(\mathrm{CaLn})_{\mathrm{N}}\left(\mathrm{Ca}_{2}\right)_{\mathrm{C}} \mathrm{CaMN60D}$. For all metals and models, the optimized lanthanide positions differ up to $1.1 \AA$ from the calcium position in the X-ray structures (Table SI6). However, both the tensors and the $Q$ factors showed only nonsignificant variations, smaller than the errors from these magnitudes (Table SI7). Since in our approach the description of the interdomain dynamics will be based on the optimization of the $Q$ factor, the lanthanide position was not further optimized during our analysis. As expected, for a protein with the paramagnetic metal in the $\mathrm{N}$-terminal domain and with anisotropic interdomain flexibility, the axial component, $\chi_{\mathrm{ax}}$ for the C-terminal tensors is between 2 and $20 \%$ smaller than for the $\mathrm{N}$-terminal tensors, and their relative orientations also deviate by up to 48,12 , and $21^{\circ}$ for the Euler angles $\alpha, \beta$, and $\gamma$, respectively. Indeed, for anisotropic domain motion, it is expected that the angles and the changes of $\chi_{\mathrm{ax}}$ of the $\mathrm{N}$ - and $\mathrm{C}$ terminal domains differ between paramagnetic ions. The reduction of the tensor size and the rotation of the relative orientations puts into evidence that none of the three X-ray models alone provides a correct description of the interdomain arrangement. Thus, if we compare the CaM/IQ complex to free
$\mathrm{CaM}^{52}$ in which the relative tensors were scaled by approximately a factor of 0.12 , our data indicate that the $\mathrm{CaM} / \mathrm{IQ}$ complex is much more rigid, but still with detectable interdomain motion, as already hinted by the presence of two different interdomain arrangements in the X-ray structure. In agreement with the dynamics shown in the crystal structure, the $\mathrm{RDC}$ and the PCS+RDC fits generally improved for the $\mathrm{N}$ - and C-terminal domains individually by using the three models $\mathrm{A}$, $\mathrm{B}$, and $\mathrm{C}$ together at equal populations and with a single alignment tensor (Figure 2). This finding indicates that the three X-ray models together are a better representation of the internal motions of each of the CaM domains than the individual models. The $Q$ values obtained were used in the following as the target values to be reached for the fitting of the ensemble of the complex.

Single Model Fails To Fully Describe the Paramagnetic Data. A previous study with paramagnetic data was performed on two similar CaM complexes (i.e., the CaM/ DAPk (death-associated protein kinase) and CaM/DRP-1 (DAPk-related protein 1)). ${ }^{61}$ In both systems, CaM is wrapped around central helices from the binding partners. The authors performed a refinement using PCSs and RDCs starting from crystal structures, which lead to structural changes both in the $\mathrm{N}$-terminal domain of $\mathrm{CaM}$ and in the interdomain arrangement. Apparently, interdomain motions were absent since all data could be fitted to a single model. In order to explore whether a single interdomain arrangement would explain our data, we performed a rigid body fit of the C-terminal coordinates by applying a translation and a rotation to it, in order to minimize the $Q$ factor. We performed this analysis to the models $\mathrm{A}, \mathrm{B}$, and $\mathrm{C}$ of our $\mathrm{CaM} / \mathrm{IQ}$ complex using the combination of the data sets from $\mathrm{Dy}, \mathrm{Tm}, \mathrm{Tb}$, and $\mathrm{Er}$, and for 

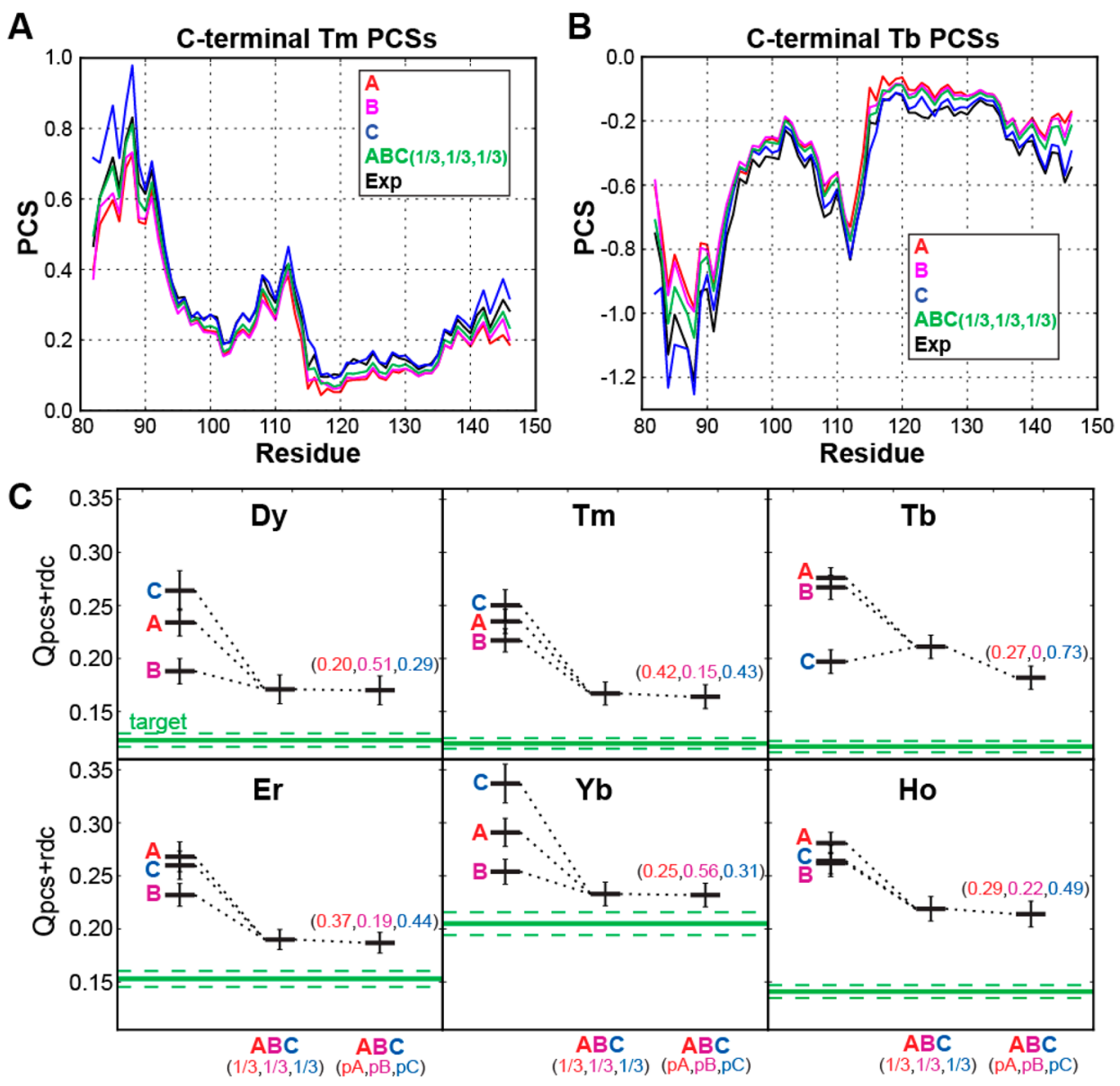

Figure 3. Evaluation of interdomain dynamics using the models A, B, and C observed in the crystal of CaM/IQ. Profiles of back-calculated Cterminal PCSs for the three X-ray models for Tm (A) and $\mathrm{Tb}(\mathrm{B})$. The experimental PCS values are shown in black, and the values for the ensemble of the three models at equal population in green. (C) Combined $Q$ factor for PCSs and RDCs from individual X-ray models (left), the ensemble of the three models at equal populations (center), and the ensemble with optimized populations (right) for each of the six lanthanides used in this study.

all models, we obtained a significant improvement of the overall $Q$ factor to values between 0.171 and 0.186 (Figure SI2). However, these values are still far from the target values defined in the previous paragraph from the fits of the individual domains, and the minimized C-terminal coordinates clash with the N-terminal CaM domain. Therefore, our analysis clearly indicates that multiple interdomain arrangements are present in the CaM/IQ complex.

X-ray Models Represent the Interdomain Dynamics to a Limited Extent. In order to study the interdomain motions, a description of the C-terminal data using the tensor from the aligned N-terminal domain is required since all PCSs and $\mathrm{RDCs}$ arise from the metals bound to the N-terminal domain. As N-terminal tensors, we used those from the PCS+RDC fits for the three X-ray models as previously described. Furthermore, as mentioned above, in order to define a target value for the $Q$ factors of anisotropic parameters of the $C$ terminal domain in the complex, we chose those obtained from individual PCS+RDC fits for the C-terminal domain. At first, we evaluated the predicted C-terminal PCSs and RDCs for the three X-ray models. An example of this analysis is shown for the PCSs from $\mathrm{Tm}$ and $\mathrm{Tb}$ (Figure 3A,B). For these metals, the model $\mathrm{C}$ provides larger PCSs than the models $\mathrm{A}$ and $\mathrm{B}$, which may be the consequence of a more compact structure in model $\mathrm{C}$, with the C-terminal atoms generally closer to the lanthanide.
Furthermore, the experimental PCSs lie within the frames defined by the models $\mathrm{A}$ and $\mathrm{C}$, resulting in a lower $Q$ factor if the models $\mathrm{A}, \mathrm{B}$, and $\mathrm{C}$ are combined with equal populations (Figure 3C). This situation was found for most of the metals, indicating that the three X-ray models better describe the conformational space than any of them separately. However, for $\mathrm{Tb}$, a different situation was found. Here, the experimental PCSs were not within the range of the predicted PCS of the three models, suggesting that additional models were required. In addition, the quality of the fits to the data from all metals did not reach the target values. Next, we explored whether the $Q$ values could be improved by variation of the populations of the models A, B, and C. The result (Figure 3C) indicated that the optimization of the populations did not provide a significant improvement of the $Q$ factors, except in the case of the $\mathrm{Tb}$ data, due to the already discussed prevalence of the model $\mathrm{C}$ in the PCSs data for this metal. Furthermore, the optimized populations were different for all metals, such that a single solution could not be found within the conformational space described by the three X-ray models, which could explain properly all the experimental data. This finding evidenced that in this complex CaM samples a larger conformational space and more models were required to depict its interdomain motions.

Incorporation of Additional Models Improves the Quality of the Fit. In order to add more conformations, we 
first evaluated the incorporation of $\mathrm{CaM}$ coordinates from other $\mathrm{CaM} /$ peptide complexes in the ensemble, by verifying their impact on the $Q$ factors. This approach was applied with the combination of the data sets from Dy, Tm, Tb, and Er. From a pool of 37 PDB structures (Table SI8), three provide significant reductions of the $Q$ factor from $0.186 \pm 0.006$ (three $\mathrm{X}$-ray models at equal populations) to values in the range of 0.164-0.165 (four X-ray models at equal population). Interestingly, all three structures belong to other CaM complexes with IQ motives from calcium channels ( $\mathrm{pdb}$ codes: $2 \mathrm{~F} 3 \mathrm{Y}^{81}{ }^{8 \mathrm{VAY}}{ }^{82}$ and $2 \mathrm{~F} 3 \mathrm{Z}^{81}$ ). The incorporation of a further PDB structure from this pool, also from a complex with an IQ motive ( $\mathrm{pdb}$ code: $3 \mathrm{BXL}$ ), ${ }^{83}$ lowered the $Q$ factors to values between 0.157 and 0.159 , but no further improvements were obtained with larger ensembles, and the target values were still not reached.

We also investigated the incorporation of one single $\mathrm{MD}$ model in the ensemble starting again from models A, B, and C and using the same metal data sets. The best MD model found using these data led to a significant reduction of the $Q$ factor from $0.186 \pm 0.006$ (three X-ray models at equal populations) to $0.151 \pm 0.005$ (three X-ray + best MD models at equal populations) (Figure 4) but still did not reach the target value for the $Q$ factor. Further improvement was achieved by adding
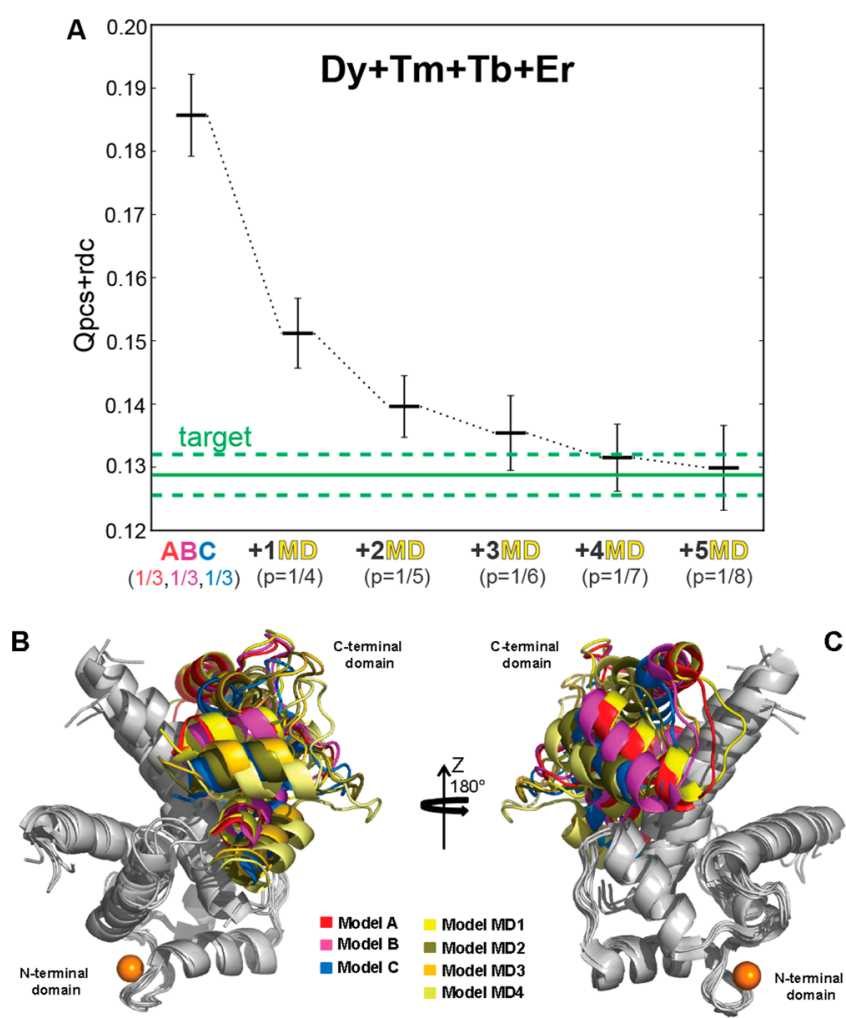

Figure 4. Impact of the incorporation of MD-derived conformations into the ensembles. (A) Dependence of the combined $Q$ factor for PCSs and RDCs for the four metals $\mathrm{Dy}-\mathrm{Tm}-\mathrm{Tb}-\mathrm{Er}$ on the ensembles (horizontal axis). The worst fit is reached with the three Xray models (left), the best ensembles including up to five MD models, all at equal populations. The ensembles with three, four, and five MD conformations reach the target value within error. Cross-validation then indicates that the ensemble with three X-ray and four MDderived conformations performs best. (B,C) Superposition of the ensemble with the four MD models onto the three X-ray structures in two views rotated by $180^{\circ}$ around the $z$-axis. additional MD models picked from the MD simulation to the ensemble as described in Materials and Methods as well as in the Supporting Information. The target value was reached within the error range when three or more MD-derived conformations were added to three X-ray structures. Similar results were obtained with ensembles made exclusively with MD models (Figure SI3), where the target value was reached also for ensembles consisting of six to eight $\mathrm{MD}$ models.

All ensembles containing MD models lead to multiple solutions that are very similar and provide similar $Q$ factors. They contain common models, as shown for the ensembles of seven structures in Tables SI10 and SI11.

Principal Component Analysis (PCA) of Interdomain Motions. In order to quantify the interdomain motions, we performed a principal component analysis of MD trajectories. The four largest eigenvectors (Figure SI4) describe more than $90 \%$ of the interdomain motions, and all combine translational and rotational motions in different directions. Not surprisingly, the projection of the pool of $37 \mathrm{PDB}$ structures of CaM complexes with different peptides along these four eigenvectors shows that they cover interdomain arrangements that are not accessible to the CaM/IQ complex (Figure SI5).

However, the four PDB structures that improved the fit when incorporated to ensembles together with models $\mathrm{A}, \mathrm{B}$, and $\mathrm{C}$ share common features. First and most importantly, they are covered by the MD trajectory, while those X-ray structures that lie outside the MD trajectory do not improve the fit. This is also important since it justifies the choice of the principal components based on the MD trajectory. In addition, the projections of these X-ray structures on eigenvectors 1 and 2 are closer to model $\mathrm{C}$ than to models $\mathrm{A}$ and $\mathrm{B}$. More importantly, three of them (i.e., 2F3Y, 2VAY, and 2F3Z) adopt more positive values along eigenvector 3, which were not assumed by model A, B, or C. Similar results were obtained for the best ensembles based on X-ray structures plus MD models (Figure SI6) as well as MD models alone (Figure SI7). They all show some extra motions along eigenvectors 1 and 2 relative to models $\mathrm{A}, \mathrm{B}$, and $\mathrm{C}$, especially the presence of some conformers that lie beyond the values from model $\mathrm{C}$. Again, they all display the same enhanced amplitude of motions along eigenvector 3 that was found in the PDB structures that provided the best solution as discussed in the paragraph before (Figure SI8). Thus, this motion that was almost missing in the models A, B, and $\mathrm{C}$, but was discovered in the $\mathrm{MD}$ simulation and also observed in the crystal structures from other CaM/IQ complexes, seems to be present in solution for our $\mathrm{CaM} / \mathrm{IQ}$ complex.

As already mentioned, our approach produced multiple solutions with similar $Q$ factors. We compared the best five solutions obtained for the sets of three X-ray plus four MD models and the corresponding sets of seven MD models (Figure SI9). The results show that they all provide a similar range of interdomain motions.

Validation of the Ensembles Describing Interdomain Motions. In order to validate these ensembles, we first evaluated the $Q$ factors for the different metals (Figure 5A). Interestingly, the ensemble including one MD model improved significantly the $Q$ factor not only for the data sets from the four metal used in the selection of this MD model but also for the data from $\mathrm{Yb}$ and Ho which were not used in the model selection but for cross-validation. In order to check whether the $\mathrm{Yb}$ and Ho tensors are linearly independent from the tensors generated by the four metals Dy, Tm, Tb, and Er used in the 

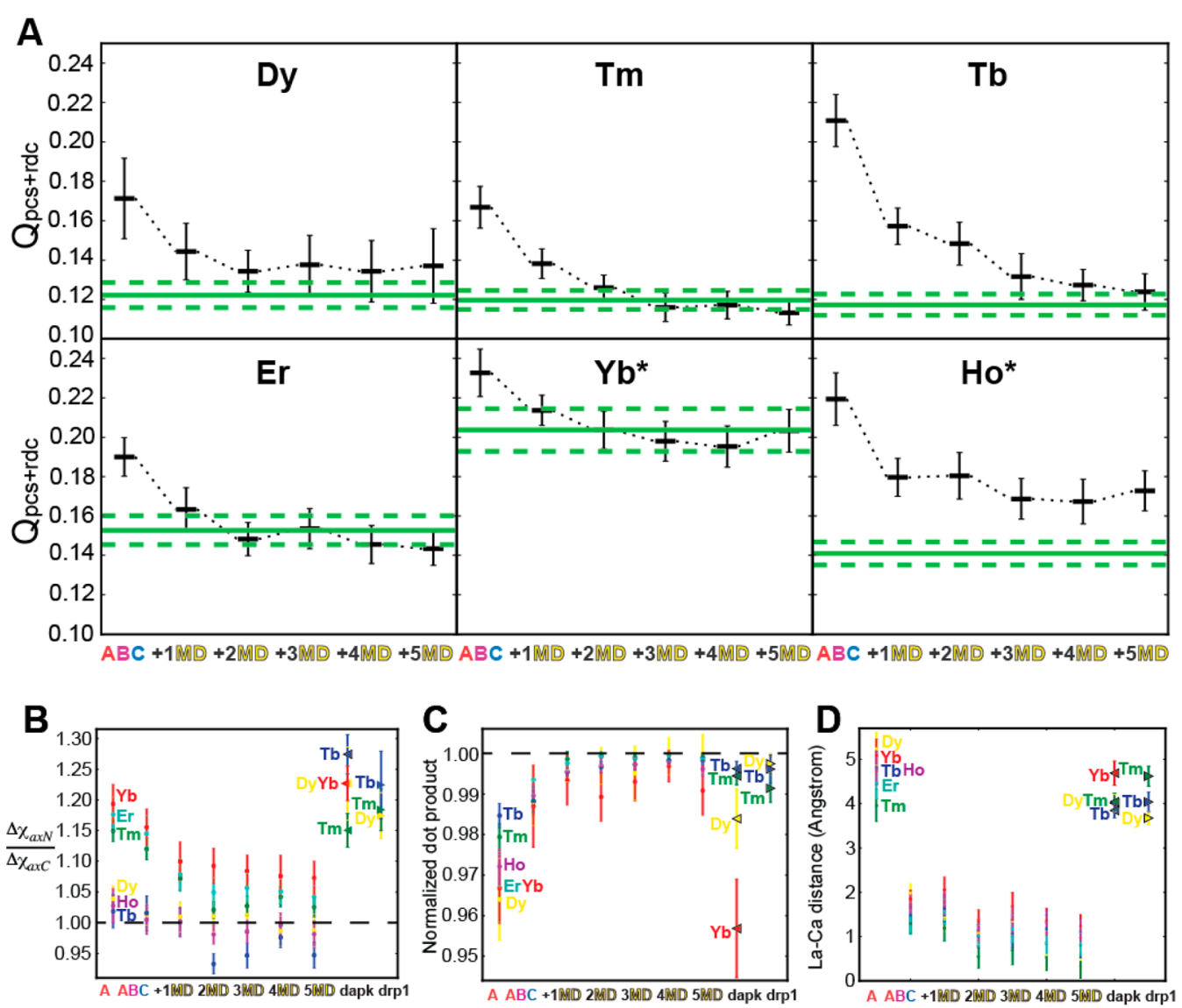

Figure 5. Validation of the different ensembles. (A) Dependence of the $Q$ values for PCS and RDC on the composition of the ensembles (horizontal axis) for the various metals. $\mathrm{Yb}$ and Ho were used for cross-validation. The increase of the $Q$ value for the ensemble with five $\mathrm{MD}$-derived conformations as compared to the ensemble with four such conformations is visible. (B,C) Comparison of $\mathrm{N}$ - and C-terminal tensors for the different ensembles (horizontal axis) with respect to the ratio of the axial components (B) and the normalized dot product of the tensors (C). The dot product is closest to 1 for the ensemble with four $\mathrm{MD}$ conformations for the two metals Ho and $\mathrm{Yb}$ that were used for cross-validation. (D) Comparison of the distance from the lanthanide position minimized using the C-terminal PCS and RDC data with the averaged calcium position in the pdb files for the different ensembles. The corresponding values for the CaM/DAPk (dapk) and CaM/DRP-1 (drp1) complexes ${ }^{61}$ are shown at the right side of the plots $(\mathrm{B}-\mathrm{D})$.

analysis, we calculated the scaled condition numbers of the following sets of five tensors, ( $\mathrm{Dy}, \mathrm{Tm}, \mathrm{Tb}, \mathrm{Er}, \mathrm{Yb}$ ) and (Dy, $\mathrm{Tm}, \mathrm{Tb}, \mathrm{Er}, \mathrm{Ho}){ }^{84}$ The scaled condition numbers are calculated from the Saupe matrices for the different metals represented as five-dimensional vectors, that is, (Szz, Sxx-Syy, Sxy, Sxz, Syz). These five elements completely describe the tensor. To calculate the scaled condition number, we first divide each Saupe vector by its norm, and then calculate the singular values for the $5 \times 5$ matrices created from the sets of five vectors. The scaled condition number is the ratio between the largest and smallest singular value. It provides a quantitative measure of how orthogonal the five Saupe vectors (i.e., tensors) are being 1 in the case of five orthogonal vectors and infinite if the vectors are linearly dependent.

These scaled condition numbers were found to be 37 and 96 for $\mathrm{Ho}$ and $\mathrm{Yb}$, respectively. While a value of 96 indicates that the holmium-derived tensor is only weakly linearly independent from the others, the condition number of 37 indicates that $\mathrm{Yb}$ is even better for cross-validation. The $Q$ factors for the different metals continued decreasing with the incorporation of up to four MD models, the data sets from Dy and Er reaching the lowest $Q$ factors already after addition of two $\mathrm{MD}$ models. The addition of the fifth $\mathrm{MD}$ model raised the $Q$ factor for Ho and
$\mathrm{Yb}$, indicating that at this point we were overfitting our data (Figure 5A). ${ }^{85}$

The similarity of $\mathrm{N}$ - and $\mathrm{C}$-terminal tensors for the different ensembles is also important in their validation since a correct ensemble is expected to deliver the same tensor for both domains of the protein. In order to quantify this similarity of the tensors derived from the $\mathrm{N}$ - and C-terminal domains, we compared the Saupe matrices. For each metal, we evaluated two parameters, the normalized dot product of the previously described $\mathrm{N}$ - and C-terminal Saupe matrices which is 1 if two tensors share the same orientation and shape, and then the ratio between the axial components, which is also 1 if the tensors have the same size. These parameters are calculated using the following equations:

$$
\text { normDot }=\frac{\vec{S}_{\mathrm{N}} \cdot \vec{S}_{\mathrm{C}}}{\left\|\vec{S}_{\mathrm{N}}\right\|\left\|\vec{S}_{\mathrm{C}}\right\|}, \quad \text { ratio }=\frac{\Delta \chi_{a x \mathrm{~N}}}{\Delta \chi_{a x \mathrm{C}}}
$$

We compared them (Table SI8) for different stages of our evaluation (Figure 5B,C): one single orientation from model A, the three $\mathrm{X}$-ray models at equal populations, and the ensembles with one to five MD models added to the X-ray structures, also at equal populations. For all metals, the normalized dot product is significantly different from the ideal value of 1 for the 
orientation given by model A (Figure 5C), which is due to the already discussed different orientation of the $\mathrm{N}$ - and C-terminal tensors for this model (Table SI4). For the ensemble with three $\mathrm{X}$-ray models, this parameter is much closer to the ideal value for all metals. The addition of MD models leads to further improvement, and the best result was reached for the set containing the three X-ray models and four MD models, where for all metals the ideal value of 1 is reached or nearly reached. On the other hand, the incorporation of the fifth MD model leads to a deviation from the ideal value for $\mathrm{Yb}$ and $\mathrm{Ho}$, which indicates overfitting of our data. A similar behavior was observed for the ratio between the axial components (Figure 5B). This parameter starts in the range from $1.02(\mathrm{~Tb})$ to 1.19 $(\mathrm{Yb})$, which means that the $\mathrm{N}$-terminal tensors are between 2 and $20 \%$ larger than the C-terminal ones. The incorporation of all X-ray models and then of MD models also made this parameter approach the ideal value for most of the data sets, also reaching the best results for the ensemble built from the three X-ray models combined with the four MD models.

Another fitting parameter when dealing with PCS data is the metal position. Thus, it is expected that a good model for interdomain motions is able to predict the actual metal position from the nontagged domain, that is, the $\mathrm{C}$-terminal domain in this study. Therefore, we compared the lanthanide positions fitted from the $\mathrm{C}$-terminal domain data with the average of the metal positions in the different ensembles (Figure 5D). Compared to the interdomain orientation in model $\mathrm{A}$, which shows deviations in the range of 3.96-5.23 $\AA$, all the ensembles with multiple models succeed in predicting the metal positions with much higher accuracy, with deviations in the range of 0.53-1.36 $\AA$ for the ensemble comprising the three X-ray and the four MD models.

We also applied our validation protocol to the refined structures of the CaM/DAPk and CaM/DRP-1 complexes. ${ }^{61}$ The main difference to our approach is that all the anisotropic data were described by a single model, thus assuming the absence of interdomain dynamics in these two CaM complexes. ${ }^{61}$ The values of the normalized dot product for the CaM/DRP-1 complex (Figure 5C) are comparable to our results in the CaM/IQ while for the CaM/DAPk complex, this parameter deviates from the ideal value for the data sets from Dy and especially from $\mathrm{Yb}$, although the values for $\mathrm{Tm}$ and $\mathrm{Tb}$ are nearly ideal. On the other hand, the ratio between the axial tensor components derived for the $\mathrm{N}$ - and the C-terminal domain lies in the range from 1.15 to 1.27 for both complexes (Figure 5B), which is a significant deviation exceeding by far the experimental errors. These structures also failed to predict the actual metal position using the C-terminal data (Figure 5D), providing values in the range of $3.68-4.69 \AA$, which are comparable to those obtained when using only the model $\mathrm{A}$ in the fit of the CaM/IQ complex.

Together, this means that although these structures (especially that of the CaM/DRP-1 complex) likely represent the average interdomain arrangement, they do not represent the actual range of interdomain motions. Probably more faithful representations of these complexes in solution would be ensembles fluctuating around the refined structures.

\section{CONCLUSIONS}

In multidomain proteins, where movements of the single domains relative to each other are of mechanistic relevance, $\mathrm{X}$ ray crystallography can be used to define the relative orientation of domains, but the conformation or conformations found in the crystal can be biased by crystal packing forces and therefore might differ from the ensemble in solution. We present here a simple approach that uses an ensemble of structures to evaluate these types of motions accurately using paramagnetic data. In this work, we mainly relied on the use of ensembles created using X-ray, MD models, and a mixture of the two and always found very similar results. Of course, this does not exclude other structural models derived, for example, from simulated annealing or Monte Carlo simulations for this type of evaluation. Even if they may provide further improvement in the analysis, our focus here was to show that ensembles of a limited number of structures reproduce faithfully experimental paramagnetic data. Our results confirm the existence of interdomain motions, as already observed in the crystal structure of the CaM/IQ complex. ${ }^{59}$ Moreover, we found that the three conformations observed in this crystal structure $^{59}$ are not sufficient to fully explain all the experimental paramagnetic data. Indeed, our results show that at least three and optimally four MD models together with the three crystal structures are required to represent the interdomain dynamics in this system. We also obtained significant improvements by adding X-ray structures from other $\mathrm{CaM} / \mathrm{IQ}$ complexes solved by crystallography, which suggests the presence of a certain pool of interdomain arrangements in this type of CaM complexes. This allowed us to identify the motions that were missing in the crystal but are present in solution.

The ensembles of structural models derived from the paramagnetic data require around 6 to 8 models for a fit that is similar to the fit of the individual domains and that is optimal in the cross-validation against unused paramagnetic data. However, ensembles of structures that are slightly different from the proposed ones but cover the same range in the hyperspace of PCAs are similarly valid (see Supporting Information).

The combined use of PCSs and RDCs is also an excellent probe to sense the rotational and translational motions that characterize interdomain dynamics. We also introduced a new practical validation scheme for models describing interdomain dynamics, which is based on the comparison of tensors derived from the domains and back-calculated metal positions. This scheme validated our ensembles and proved that the single model solution applied to similar CaM complexes ${ }^{61}$ fails to describe accurately the interdomain motions.

Our finding that domain motion in solution exceeds the one in crystals is in good agreement with the results of a recent study, ${ }^{86}$ which shows, using contrast matched SAXS/WAXS and $\mathrm{RDC}$ data, that both X-ray crystallography and standard NMR techniques fail to provide a proper picture of the interdomain orientation in CaM complexes. In the light of our results and those from Grishaev et al., ${ }^{86}$ the question arises whether all CaM complexes with helical peptides share a certain range of interdomain motions, which generally cannot be probed by standard NMR techniques or crystallography but requires approaches like the one here to derive ensembles with atomic resolution.

\section{ASSOCIATED CONTENT}

\section{Supporting Information}

Protocol for incorporation of MD models in the evaluation of interdomain dynamics; experimental PCS and RDC data; independent fits of the data to the CaM domains, with magnetic susceptibility anisotropy tensors and $Q$ factors; optimized metal positions, tensors, and $Q$ factors with or 
without lanthanide optimization; impact on the $Q$ factor of rigid body minimization of the $\mathrm{C}$-terminal domain coordinates; analysis of interdomain dynamics using MD-derived ensembles; PCA analysis of the structure ensembles; magnetic susceptibility anisotropy tensors and $Q$ factors for different ensembles; quality check of the four MD models from the optimal $A B C$ $+4 \mathrm{MD}$ ensemble. This material is available free of charge via the Internet at http://pubs.acs.org.

\section{AUTHOR INFORMATION}

\section{Corresponding Author}

cigr@nmr.mpibpc.mpg.de

\section{Author Contributions}

${ }^{\dagger}$ These authors contributed equally.

\section{Notes}

The authors declare no competing financial interest.

\section{ACKNOWLEDGMENTS}

This work was supported by the Max Planck Society. We thank Filip Van Petegem and Daniel L. Minor Jr. for the GST-IQ domain fusion protein construct initially used in this study. We thank Yvonne Laukat for technical help. This work was supported by BioNMR (grant: EU 261863).

\section{REFERENCES}

(1) Sicheri, F.; Kuriyan, J. Curr. Opin. Struct. Biol. 1997, 7, 777-85.

(2) Pickford, A. R.; Campbell, I. D. Chem. Rev. 2004, 104, 3557-66.

(3) Zhang, Y.; Zuiderweg, E. R. Proc. Natl. Acad. Sci. U.S.A. 2004, $101,10272-7$

(4) Tonks, N. K.. Nat. Rev. Mol. Cell Biol. 2006, 7, 833-46.

(5) Chuang, G. Y.; Mehra-Chaudhary, R.; Ngan, C. H.; Zerbe, B. S.; Kozakov, D.; Vajda, S.; Beamer, L. J. Protein Sci. 2010, 19, 1662-72.

(6) Gerstein, M.; Krebs, W. Nucleic Acids Res. 1998, 26, 4280-90.

(7) Shilton, B. H.; Flocco, M. M.; Nilsson, M.; Mowbray, S. L. J. Mol. Biol. 1996, 264, 350-63.

(8) Zhang, X. J.; Wozniak, J. A.; Matthews, B. W. J. Mol. Biol. 1995, 250, 527-52.

(9) Faber, H. R.; Matthews, B. W. Nature 1990, 348, 263-6.

(10) Bruschweiler, R. Curr. Opin. Struct. Biol. 2003, 13, 175-83.

(11) Eisenmesser, E. Z.; Bosco, D. A.; Akke, M.; Kern, D. Science 2002, 295, 1520-3.

(12) Mulder, F. A.; Mittermaier, A.; Hon, B.; Dahlquist, F. W.; Kay,

L. E. Nat. Struct. Biol. 2001, 8, 932-5.

(13) Ryabov, Y. E.; Fushman, D. J. Am. Chem. Soc. 2007, 129, 331527.

(14) Baber, J. L.; Szabo, A.; Tjandra, N. J. Am. Chem. Soc. 2001, 123, 3953-9.

(15) Tolman, J. R.; Al-Hashimi, H. M.; Kay, L. E.; Prestegard, J. H. J. Am. Chem. Soc. 2001, 123, 1416-24.

(16) Meiler, J.; Prompers, J. J.; Peti, W.; Griesinger, C.; Bruschweiler, R. J. Am. Chem. Soc. 2001, 123, 6098-107.

(17) Barbato, G.; Ikura, M.; Kay, L. E.; Pastor, R. W.; Bax, A. Biochemistry 1992, 31, 5269-78.

(18) Ishima, R.; Torchia, D. A. Nat. Struct. Biol. 2000, 7, 740-3.

(19) Lindorff-Larsen, K.; Best, R. B.; Depristo, M. A.; Dobson, C. M. Nature 2005, 433, 128-32.

(20) Chang, S. L.; Tjandra, N. J. Am. Chem. Soc. 2001, 123, 11484-5.

(21) Chen, K.; Tjandra, N. J. Am. Chem. Soc. 2008, 130, 12745-51.

(22) Ryabov, Y.; Clore, G. M.; Schwieters, C. D. J. Chem. Phys. 2012, 136, 034108.

(23) Wong, V.; Case, D. A.; Szabo, A. Proc. Natl. Acad. Sci. U.S.A. 2009, 106, 11016-21.

(24) Horne, J.; d'Auvergne, E. J.; Coles, M.; Velkov, T.; Chin, Y.; Charman, W. N.; Prankerd, R.; Gooley, P. R.; Scanlon, M. J. J. Mol. Biol. 2007, 371, 703-16.
(25) Yuwen, T.; Post, C. B.; Skrynnikov, N. R. J. Biomol. NMR 2011, $51,131-50$

(26) Martín-Pastor, M.; Canales, A.; Corzana, F.; Asensio, J. L.; Jiménez-Barbero, J. J. Am. Chem. Soc. 2005, 127, 3589-95.

(27) Erdélyi, M.; d'Auvergne, E.; Navarro-Vázquez, A.; Leonov, A.; Griesinger, C. Chemistry 2011, 17, 9368-76.

(28) Yamamoto, S.; Yamaguchi, T.; Erdélyi, M.; Griesinger, C.; Kato, K. Chemistry 2011, 17, 9280-2.

(29) Sun, H.; Reinscheid, U. M.; Whitson, E. L.; d'Auvergne, E. J.; Ireland, C. M.; Navarro-Vázquez, A.; Griesinger, C. J. Am. Chem. Soc. 2011, 133, 14629-36.

(30) Bertini, I.; Luchinat, C.; Parigi, G. Prog. NMR Spectrosc. 2002, 40, 249-73.

(31) Gaponenko, V.; Altieri, A. S.; Li, J.; Byrd, R. A. J. Biomol. NMR 2002, 24, 143-8.

(32) Ikegami, T.; Verdier, L.; Sakhaii, P.; Grimme, S.; Pescatore, B.; Saxena, K.; Fiebig, K. M.; Griesinger, C. J. Biomol. NMR 2004, 29, 339-49.

(33) Leonov, A.; Voigt, B.; Rodriguez-Castañeda, F.; Sakhaii, P.; Griesinger, C. Chemistry 2005, 11, 3342-8.

(34) Haberz, P.; Rodriguez-Castañeda, F.; Junker, J.; Becker, S.; Leonov, A. Org. Lett. 2006, 8, 1275-8.

(35) Rodriguez-Castañeda, F.; Haberz, P.; Leonov, A.; Griesinger, C. Magn. Reson. Chem. 2006, 44, S10-6.

(36) Peters, F.; Maestre-Martinez, M.; Leonov, A.; Kovačič, L.; Becker, S.; Boelens, R.; Griesinger, C. J. Biomol. NMR 2011, 51, 32937.

(37) Liu, W. M.; Keizers, P. H.; Hass, M. A.; Blok, A.; Timmer, M.; Sarris, A. J.; Overhand, M.; Ubbink, M. J. Am. Chem. Soc. 2012, 134, 17306-13.

(38) Su, X. C.; Man, B.; Beeren, S.; Liang, H.; Simonsen, S.; Schmitz, C.; Huber, T.; Messerle, B. A.; Otting, G. J. Am. Chem. Soc. 2008, 130, 10486-7.

(39) Man, B.; Su, X. C.; Liang, H.; Simonsen, S.; Huber, T.; Messerle, B. A.; Otting, G. Chemistry 2010, 16, 3827-32.

(40) Swarbrick, J. D.; Ung, P.; Su, X. C.; Maleckis, A.; Chhabra, S.; Huber, T.; Otting, G.; Graham, B. Chem. Commun. 2011, 47, 736870.

(41) Hansen, A. L.; Al-Hashimi, H. M. J. Am. Chem. Soc. 2007, 129, $16072-82$.

(42) Martin, L. J.; Hähnke, M. J.; Nitz, M.; Wöhnert, J.; Silvaggi, N. R; Allen, K. N.; Schwalbe, H.; Imperiali, B. J. Am. Chem. Soc. 2007, 129, 7106-13.

(43) Jia, X.; Yagi, H.; Su, X. C.; Stanton-Cook, M.; Huber, T.; Otting, G. J. Biomol. NMR 2011, 50, 411-20.

(44) Graham, B.; Loh, C. T.; Swarbrick, J. D.; Ung, P.; Shin, J.; Yagi, H.; Jia, X.; Chhabra, S.; Barlow, N.; Pintacuda, G.; Huber, T.; Otting, G. Bioconjugate Chem. 2011, 22, 2118-25.

(45) Li, Q. F.; Yang, Y.; Maleckis, A.; Otting, G.; Su, X. C. Chem. Commun. 2012, 48, 2704-6.

(46) Keizers, P. H.; Ubbink, M. Prog. Nucl. Magn. Reson. Spectrosc. 2011, 58, 88-96.

(47) Keizers, P. H.; Saragliadis, A.; Hiruma, Y.; Overhand, M.; Ubbink, M. J. Am. Chem. Soc. 2008, 130, 14802-12.

(48) Hass, M. A.; Keizers, P. H.; Blok, A.; Hiruma, Y.; Ubbink, M. J. Am. Chem. Soc. 2010, 132, 9952-3.

(49) Feeny, J.; Birdsall, B.; Bradbury, A. F.; Biekofsky, R. R.; Bayley, P. M. J. Biomol. NMR 2001, 21, 41-8.

(50) Wöhnert, J.; Franz, K. J.; Nitz, M.; Imperiali, B.; Schwalbe, H. J. Am. Chem. Soc. 2003, 125, 13338-9.

(51) Silvaggi, N. R.; Martin, L. J.; Schwalbe, H.; Imperiali, B.; Allen, K. N. J. Am. Chem. Soc. 2007, 129, 7114-20.

(52) Bertini, I.; Del Bianco, C.; Gelis, I.; Katsaros, N.; Luchinat, C.; Parigi, G.; Peana, M.; Provenzani, A.; Zoroddu, M. A. Proc. Natl. Acad. Sci. U.S.A. 2004, 101, 6841-6.

(53) Dasgupta, S.; Hu, X.; Keizers, P. H.; Liu, W. M.; Luchinat, C.; Nagulapalli, M.; Overhand, M.; Parigi, G.; Sgheri, L.; Ubbink, M. J. Biomol. NMR 2011, 51, 253-63. 
(54) Frank, A. T.; Stelzer, A. C.; Al-Hashimi, H. M.; Andricioaei, I. Nucleic Acids Res. 2009, 37, 3670-9.

(55) Zhang, Q.; Stelzer, A. C.; Fisher, C. K.; Al-Hashimi, H. M. Nature 2007, 450, 1263-7.

(56) Bertini, I.; Gupta, Y. K.; Luchinat, C.; Parigi, G.; Peana, M.; Sgheri, L.; Yuan, J. J. Am. Chem. Soc. 2007, 129, 12786-94.

(57) Bertini, I.; Giachetti, A.; Luchinat, C.; Parigi, G.; Petoukhov, M. V.; Pierattelli, R.; Ravera, E.; Svergun, D. I. J. Am. Chem. Soc. 2010, 132, 13553-8.

(58) Longinetti, M.; Luchinat, C.; Parigi, G.; Sgheri, L. Inverse Probl. 2006, 22, 17.

(59) Van Petegem, F.; Chatelain, F. C.; Minor, D. L. Nat. Struct. Mol. Biol. 2005, 12, 1108-15.

(60) Bertini, I.; Gelis, I.; Katsaros, N.; Luchinat, C.; Provenzani, A. Biochemistry 2003, 42, 8011-21.

(61) Bertini, I.; Kursula, P.; Luchinat, C.; Parigi, G.; Vahokoski, J.; Wilmanns, M.; Yuan, J. J. Am. Chem. Soc. 2009, 131, 5134-44.

(62) Guerini, D.; Krebs, J.; Carafoli, E. J. Biol. Chem. 1984, 259, $15172-7$.

(63) Bogomolovas, J.; Simon, B.; Sattler, M.; Stier, G. Protein Expression Purif. 2009, 64, 16-23.

(64) Kay, L. E.; Xu, G. Y.; Yamazaki, T. J. Magn. Reson. 1994, A 109, 129-33.

(65) Schleucher, J.; Sattler, M.; Griesinger, C. Angew. Chem., Int. Ed. Engl. 1993, 32, 1489-91.

(66) Grzesiek, S.; Bax, A. J. Magn. Reson. 1992, 96, 432-40.

(67) Grzesiek, S.; Bax, A. J. Biomol. NMR 1993, 3, 185-204.

(68) Ottiger, M.; Delaglio, F.; Bax, A. J. Magn. Reson. 1998, 131, 373-8.

(69) Delaglio, F.; Grzesiek, S.; Vuister, G. W.; Zhu, G.; Pfeifer, J.; Bax, A. J. Biomol. NMR 1995, 6, 277-93.

(70) Goddard, T. D.; Kneller, D. G. SPARKY 3; University of California, San Francisco, 2008.

(71) Keller, R.. CARA; Zürich, 2004.

(72) Hornak, V.; Abel, R.; Okur, A.; Strockbine, B.; Roitberg, A. Proteins 2006, 65, 712-25.

(73) Hess, B.; Kutzner, C.; van der Spoel, D.; Lindahl, E. J. Chem. Theory Comput. 2008, 4, 435-447.

(74) DeLano, W. L. The PyMOL Molecular Graphics System (2002); DeLano Scientific: San Carlos, CA, 2002.

(75) Laskowski, R. A.; Rullmannn, J. A.; MacArthur, M. W.; Kaptein, R.; Thornton, J. M. J. Biomol. NMR 1996, 8, 477-86.

(76) Davis, I. W.; Leaver-Fay, A.; Chen, V. B.; Block, J. N.; Kapral, G. J.; Wang, X.; Murray, L. W.; Arendall, W. B.; Snoeyink, J.; Richardson, J. S.; Richardson, D. C. Nucleic Acids Res. 2007, 35, W375-83.

(77) Cornilescu, G.; Ottiger, M.; Bax, A. J. Am. Chem. Soc. 1998, 120, 6836-7.

(78) Bentrop, D.; Bertini, I.; Cremonini, M. A.; Forsén, S.; Luchinat, C.; Malmendal, A. Biochemistry 1997, 36, 11605-18.

(79) Frederick, K. K.; Marlow, M. S.; Valentine, K. G.; Wand, A. J. Nature 2007, 448, 325-9.

(80) Fischer, M. W.; Losonczi, J. A.; Weaver, J. L.; Prestegard, J. H. Biochemistry 1999, 38, 9013-22.

(81) Fallon, J. L.; Halling, D. B.; Hamilton, S. L.; Quiocho, F. A. Structure 2005, 13, 1881-6.

(82) Halling, D. B.; Georgiou, D. K.; Black, D. J.; Yang, G.; Fallon, J. L.; Quiocho, F. A.; Pedersen, S. E.; Hamilton, S. L. J. Biol. Chem. 2009, 284, 20041-51.

(83) Mori, M. X.; Vander Kooi, C. W.; Leahy, D. J.; Yue, D. T. Structure 2008, 16, 607-20.

(84) Peti, W.; Meiler, J.; Brüschweiler, R.; Griesinger, C. J. Am. Chem. Soc. 2002, 124, 5822-33.

(85) Bonvin, A. M.; Brünger, A. T. J. Mol. Biol. 1995, 250, 80-93.

(86) Grishaev, A.; Anthis, N. J.; Clore, G. M. J. Am. Chem. Soc. 2012, $134,14686-9$. 